\title{
Investigation of the elimination process of a multimodal polymer-shelled contrast agent in rats using ultrasound and transmission electron microscopy
}

\author{
Johan Härmark $^{\mathrm{a}, *}$, Malin K. Larsson ${ }^{\mathrm{b}}$, Anton Razuvajev ${ }^{\mathrm{c}}$, Philip J.B. Koeck ${ }^{\mathrm{a}}$, \\ Gaio Paradossi ${ }^{\mathrm{d}}$, Lars-Åke Brodin ${ }^{\mathrm{b}}$, Kenneth Caidahl ${ }^{\mathrm{c}}$, Hans Hebert ${ }^{\mathrm{a}}$ and Anna Bjällmark ${ }^{\mathrm{b}, \mathrm{c}}$

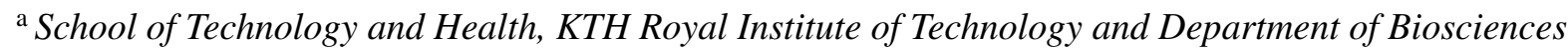 \\ and Nutrition, Karolinska Institutet, Stockholm, Sweden \\ ${ }^{\mathrm{b}}$ Department of Medical Engineering, School of Technology of Health, KTH Royal Institute of \\ Technology, Stockholm, Sweden \\ ${ }^{\mathrm{c}}$ Department of Molecular Medicine and Surgery, Karolinska Institutet, Stockholm, Sweden \\ ${ }^{\mathrm{d}}$ Department of Chemical Sciences and Technologies, Università di Roma Tor Vergata, Rome, Italy
}

\begin{abstract}
.
BACKGROUND: A novel polymer-shelled contrast agent (CA) with multimodal imaging and target specific potential was developed recently and tested for its acoustical properties using different in-vitro setups.

OBJECTIVE: The aim of this study was to investigate the elimination of three types of the novel polymer-shelled CA, one unmodified and two shell modified versions, in rats.

METHODS: The blood elimination time was estimated by measuring the image intensity, from ultrasound images of the common carotid artery, over time after a bolus injection of the three types of the novel CA. The commercially available CA SonoVue was used as a reference. The subcellular localization of the three CAs was investigated using transmission electron microscopy.

RESULTS: The ultrasound measurements indicated a blood half-life of 17-85 s for the different types of the novel CA, which was significant longer than the blood half-life time for SonoVue. Additionally, CAs were exclusively found in the circulatory system, either taken up by, or found in the vicinity of macrophages.

CONCLUSIONS: Compared to the commercially available CA SonoVue, the blood circulation times for the three types of the novel polymer-shelled CA were prolonged. Moreover, macrophages were suggested to be responsible for the elimination of the CA.
\end{abstract}

Keywords: Contrast agent, polymer, subcellular localization, transmission electron microscope, ultrasound imaging

\section{Background}

Multimodality imaging is a promising approach in the diagnosis of various diseases as it could provide both anatomical and physiological information when combining two or more imaging modalities, such

\footnotetext{
${ }^{*}$ Corresponding author: Johan Härmark, School of Technology and Health, KTH Royal Institute of Technology, Alfred Nobels Allé 10, 14152 Huddinge, Sweden. Tel.: +46 8524810 91; Fax: +46 821 83 68; E-mail: jharmark@kth.se.
} 
as ultrasound and magnetic resonance imaging (MRI). To further increase the sensitivity and specificity of multimodal imaging, contrast agents (CAs) consisting of 2-6 $\mu \mathrm{m}$ sized encapsulated microbubbles (MBs) supporting multimodal imaging would be of great value. A novel polymer-shelled CA with multimodal and target specific potential was developed recently. This CA consists of monodisperse air filled MBs and has demonstrated a long shelf life [4]. Moreover, the shell surface enables the coating of different molecules [5]. In order to serve as a CA for ultrasound and MRI, the shell of the MBs was decorated with superparamagnetic iron oxide nanoparticles (SPIONs) [3]. Thin sectioning transmission electron microscopy (TEM) has been shown to be of great value for determining the structure of these and other polymer-shelled particles [3,11].

Micrometer-sized particles are pure blood pool agents as their size means that they will not be able to pass between the endothelial cells [14]. It has been demonstrated that CAs have a blood half-life ranging from minutes to hours $[6,12,15,22,23]$. Variations in blood half-life can be explained by different shell structure, size and animal model. Most studies evaluating blood elimination time utilize radioactivity measurements or gas analysis $[6,12,15,22,23]$, but a different approach could be to study ultrasound signal intensity decay, as the signal intensity corresponds to the concentration of MBs [18].

Depending on the animal model investigated, different organ accumulations of CA have been observed, when using a CA stabilized with an albumin shell [24]. The presence of pulmonary macrophages in pigs was thought to be responsible for their high uptake of CA in the lungs. In contrast, when using a rat model, the predominant part of the dose was observed in the liver shortly after injection, which was most probably due to the lack of specific pulmonary macrophages. By studying the biodistribution of iodine-labeled CAs in a rat model, it was concluded that the CA uptake in the liver was mainly performed by the macrophages [16]. Comparable results were also observed during histological analysis of CA uptake in rat liver cells utilizing TEM [17]. Studies of liposomes, which are comparable to the lipidshell CAs in terms of surface properties, have shown that the subcellular distribution was size-dependent, and that liposomes with a diameter larger than $200 \mathrm{~nm}$ were mainly phagocytosed by macrophages [14]. Polymer-shelled CAs, having a completely different external surface, might have a unique pattern of cell interaction resulting in different elimination times compared with other CAs. Therefore, it is essential to study the subcellular localization of this novel polymer-shelled CA.

The research comprising this novel polymer-shelled CA has so far mainly been focused on in-vitro experiments, showing its potential to serve as multimodal CA $[3,8,9,19]$. Prior to initiation of extensive in-vivo studies, the interaction with biological material must be determined. The aim of this study was therefore to investigate the elimination of three types of the polymer-shelled CA; one unmodified polymer-shelled CA (Plain MB) useful solely with ultrasound imaging and two shell-modified CAs (Type A and Type B) for combined ultrasound and magnetic resonance imaging. The elimination profile was investigated using a rat model by measuring the ultrasound intensity over time and by analyzing tissue sections using TEM.

\section{Methods}

This study comprised 25 rats and 3 types of the novel polymer-shelled CA. The blood elimination time was assessed by analyzing the image intensity obtained from ultrasound images of the common carotid artery. In addition, the commercially available CA SonoVue (Bracco Imaging, Italy) was used as a reference during the blood elimination time measurements. As a complement to these measurements, the sensitivity of the ultrasound system, in terms of the lowest detectable concentration of CA, was 
Table 1

The contrast agents and the number of animals included in the study

\begin{tabular}{lcccc}
\hline Contrast agent & \multicolumn{3}{c}{ Histological analysis (number of rats) } & Ultrasound imaging (number of rats) \\
\cline { 2 - 5 } & 10 min & 40 min & $24 \mathrm{~h}$ & - \\
\hline Plain MB $\left(7.5 \times 10^{7} \mathrm{MBs} / \mathrm{ml}\right)$ & 1 & 1 & 1 & - \\
Type A $\left(7.5 \times 10^{7} \mathrm{MBs} / \mathrm{ml}\right)$ & 1 & 1 & 1 & - \\
Type B $\left(7.5 \times 10^{7} \mathrm{MBs} / \mathrm{ml}\right)$ & 1 & 1 & 1 & 4 \\
Plain MB $\left(6.6 \times 10^{8} \mathrm{MBs} / \mathrm{ml}\right)$ & - & - & - & 4 \\
Type A $\left(6.6 \times 10^{8} \mathrm{MBs} / \mathrm{ml}\right)$ & - & - & - & 4 \\
Type B $\left(6.6 \times 10^{8} \mathrm{MBs} / \mathrm{ml}\right)$ & - & - & - & 4 \\
SonoVue $\left(2-5 \times 10^{8} \mathrm{MBs} / \mathrm{ml}\right)$ & - & - & - & 4 \\
\hline
\end{tabular}

Notes: MB - microbubble, ${ }^{*}$ tissue samples from liver, spleen, kidney and lungs were analyzed for each animal, ${ }^{\dagger}$ two image sequences were acquired for each animal.

investigated in an in-vitro setup. Moreover, the interaction of $\mathrm{CA}$ at a subcellular level in the liver, spleen, kidney and lung at different time points after CA injection was evaluated utilizing TEM.

\subsection{Animals}

The study protocol (N372/10) was approved by the local ethical committee of animal experiments in Solna, Sweden. A total of 25 rats were included in this study. In the ultrasound imaging experiment, 16 rats $(\sim 450 \mathrm{~g})$ were included while the histology analysis comprised 9 rats $(\sim 450 \mathrm{~g})$, see Table 1 . All the rats were anesthetized utilizing 3-4\% isoflurane, and to maintain anesthesia, 1-2\% isoflurane was given. During anesthesia, the body temperature was controlled by a heating pad. The right jugular vein was catheterized. The rats, in which histological analysis was performed after $24 \mathrm{~h}$, were given a subcutaneously dose of temgesic $(0.01 \mathrm{mg} / \mathrm{kg}$ body weight $)$ for pain relief prior to and $12 \mathrm{~h}$ after the surgery. Acute experiments were carried out in the remaining cases.

\subsection{Contrast agents}

Three types of the in-house made polymer-shelled CA were employed in the study (Table 1); one unmodified polymer-shelled CA (Plain MB) designed for ultrasound imaging [4] and two shell modified CAs, both containing SPIONs for combined ultrasound and magnetic resonance imaging [3], see Fig. 1. Plain MB are produced by cross-linking of synthesized polyvinyl alcohol at the air-water interface during high-shear stirring with an Ultra Turrax (IKA, Germany) stirrer at $8000 \mathrm{rpm}$ [4]. In one of the modified CAs, the SPIONs were covalently linked to the polymer shell surface via chitosan molecules (Type A), whereas in the other modified CA, the SPIONs were embedded within the shell (Type B) [3]. Moreover, SonoVue was used as a reference in the ultrasound experiments. The diameter was $3.8 \pm 0.6 \mu \mathrm{m}$ [3] for the three types of the polymer-shelled CA and $2.5 \mu \mathrm{m}$ (range: 1-10 $\mu \mathrm{m}$ ) [7] in diameter for SonoVue.

A bolus injection of $0.4 \mathrm{ml}$ of the CAs listed in Table 1 was manually injected through the right jugular vein of the rats, followed by a flush of saline. The injection flow was approximately $0.03 \mathrm{ml} / \mathrm{s}$. Two repeated injections of the same CA were performed in the animals exposed to ultrasound.

\subsection{Ultrasound imaging}

\subsubsection{In-vitro: Lowest detectable concentration of contrast agent}

In order to determine the sensitivity of the ultrasound system for detecting CA, a tissue mimicking flow phantom with a vessel diameter of $6 \mathrm{~mm}$ was designed [19]. Dilution series $\left(10^{2}, 10^{3}, 10^{4}, 10^{5}\right.$, 

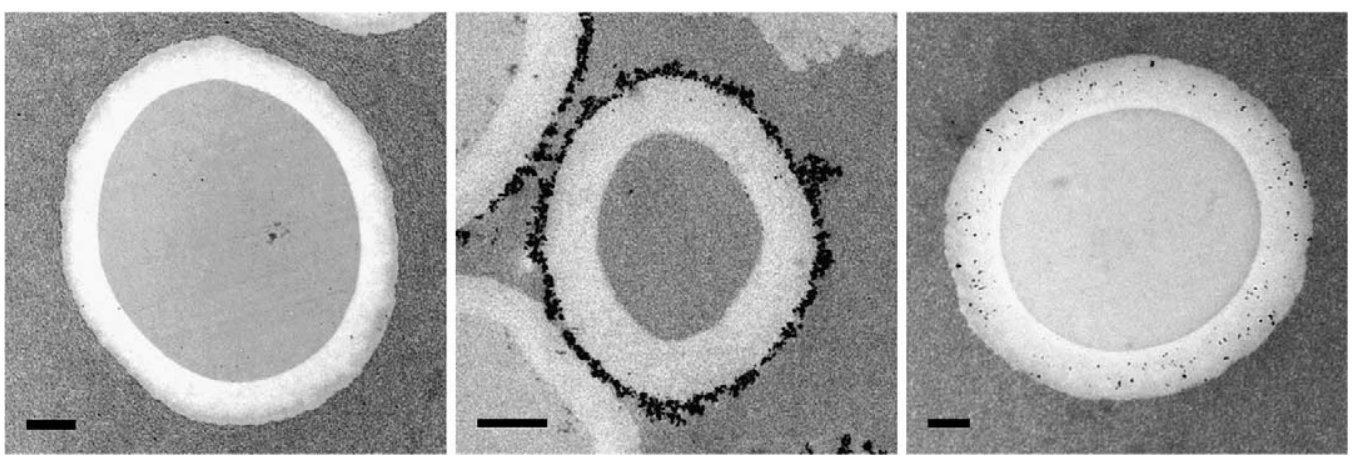

Fig. 1. TEM images showing cross sections of the three types of CAs. From left: Plain MB, Type A with SPIONs (dark dots) covalently bound to the surface of the MBs and Type B with SPIONs (dark dots) embedded inside the MB shell. Note that the cross sections were obtained at random distances from the equatorial plane of the MBs, resulting in size difference between the analyzed MBs. Scale bars represent $500 \mathrm{~nm}$.

$10^{6} \mathrm{MBs} / \mathrm{ml}$ ) were prepared by mixing stock solutions of both Plain MB and SonoVue with de-ionized water to desired concentrations. The solution was constantly stirred by a magnetic stirrer and transported through the tissue mimicking flow phantom at a mean flow of $1.8 \mathrm{ml} / \mathrm{s}$ by using a peristaltic pump (Watson Marlow, United Kingdom), see Fig. 2(a). A high frequency ultrasound system, Vevo 2100 (frequency $=18 \mathrm{MHz}$, frame rate $=15$ frames/s) (FUJIFILM VisualSonics Inc., Canada), in the nonlinear contrast mode based on power modulation was used. Before image acquisition was initiated, the probe (MS250, center frequency $=21 \mathrm{MHz}$ ) was fixed in a position over the phantom by using a tripod holder. Moreover, the focus point was placed in the middle of the mimicked vessel lumen and gain was individually optimized for each image sequence. Thereafter, ultrasound short-axis image sequences ( $n=260$ ) were acquired, starting with the lowest concentration of the CAs tested. A circulating solution of pure de-ionized water was imaged prior to each dilution series, serving as control sequences. The mean intensity within a region of interest (ROI), with an area of $15 \mathrm{~mm}^{2}$, placed in the middle of the vessel lumen was investigated for each dilution and CA by using the custom-made workstation, Vevo software (FUJIFILM VisualSonics Inc., Canada), see Fig. 2(b).

By comparing the mean intensity obtained during control imaging $\left(\mathrm{ROI}_{\text {control }}\right)$ using pure de-ionized water with each $\mathrm{CA}$ dilution $\left(\mathrm{ROI}_{\text {dilution }}\right)$, the lowest detectable concentration of $\mathrm{CA}$ was determined. A detectable concentration for the present ultrasound system was assumed if a significantly higher intensity was observed for $\mathrm{ROI}_{\text {dilution }}(n=260)$ compared with the intensity in $\mathrm{ROI}_{\text {control }}(n=260)$. This was tested with a $z$-test at a significance level of $5 \%$.

\subsubsection{In-vivo: Blood elimination time measurements}

Ultrasound image sequences (1.5-2 min) of the left common carotid artery in the long-axis view were continuously acquired for $10 \mathrm{~min}$ after the CA injection or shorter if the signal enhancements in the vessel lumen was no longer visually detectable, see Fig. 3. The second injection of CA was performed $30 \mathrm{~min}$ after the first injection in each rat. The same imaging system and settings as previously described in the in-vitro experiments were used. The employed transducer was fixed by a tripod holder on the left common carotid artery and the focus point was fixed in the middle of the vessel lumen.

After completed ultrasound acquisition, the image sequences were exported to a workstation, Vevo software (FUJIFILM VisualSonics Inc., Canada), for offline analysis. The image sequences were excluded if movements of the animal resulted in a shift of the image view within the stored image sequences. A ROI with an area of approximately $1.5 \mathrm{~mm}^{2}$ was manually placed in the middle of the vessel 
A

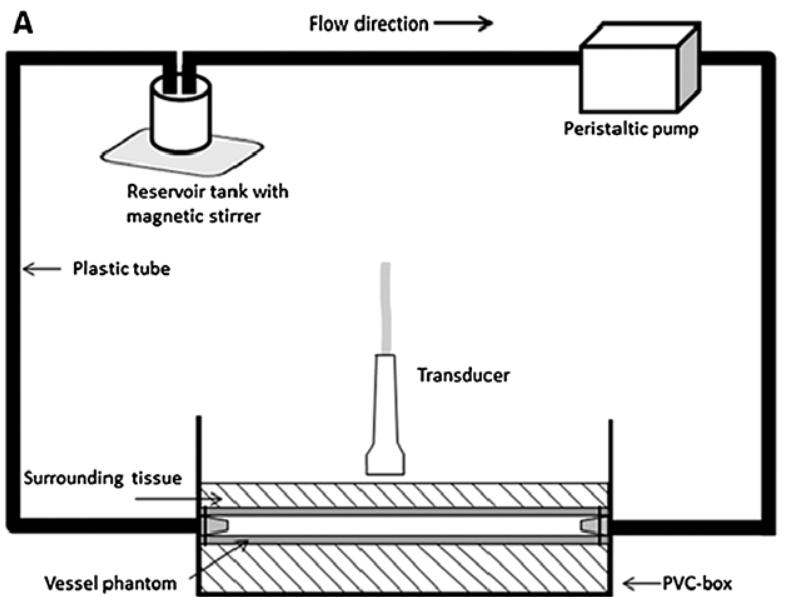

B

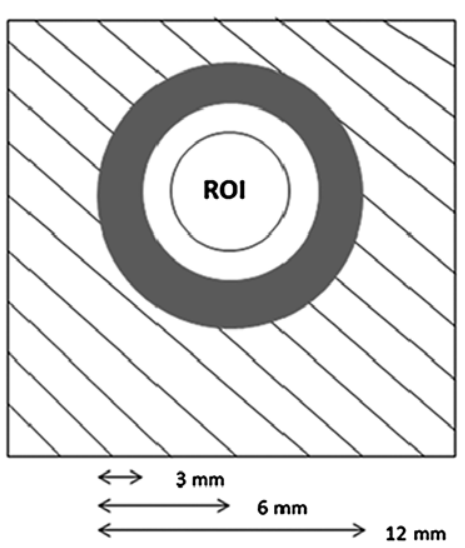

Fig. 2. The in-vitro setup and phantom vessel dimension. (A) Schematic illustration of the in-vitro setup. (B) Illustration of the vessel dimension of the tissue mimicking phantom combined with the region of interest used for the intensity measurements.
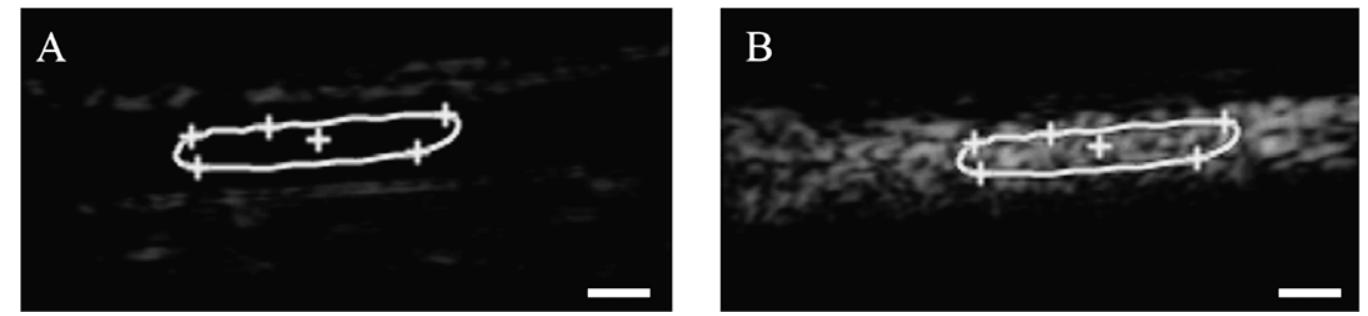

Fig. 3. Ultrasound images of the common carotid artery of a rat. Ultrasound images obtained in the long axis view of the common carotid artery of a rat, before (A) and after (B) injection of Type B contrast agent. The mean intensity within the region of interest (ROI) is exported for off line analysis. Scale bars represents $1 \mathrm{~mm}$.

lumen (Fig. 3) and the mean intensity within the ROI from each frame was exported from the workstation and further analyzed using MATLAB (MathWorks, USA). The data were then preprocessed with a noise reduction filter by convolving the intensity curves with a normalized rectangular function of 8 time samples in length. Thereafter, in order to assess the wash-out curves for the different CAs, normalized curves (starting point $=$ peak intensity) were approximated to an exponential decay curve using the nonlinear least square method. The median blood elimination time for each CA was determined. The differences in elimination between the CAs were evaluated using a Mann-Whitney U-test (5\% significance level).

\subsection{Histological analysis by transmission electron microscopy}

Tissue samples from liver, spleen, kidney and lung were collected at different time points (10 min, $40 \mathrm{~min}$ and $24 \mathrm{~h}$ ) after injection and cut into $1 \mathrm{~mm}$ slices, see Table 1 . Regions $\left(1 \mathrm{~mm}^{2}\right)$ containing parenchyma and endothelial cells, e.g. the liver hepatocytes, were randomly selected for liver, spleen and lung tissue samples. On the contrary, the objective for the kidney was to investigate if the CA had passed the filtration barrier between blood and urine in the glomerulus. As a consequence, tissue samples $\left(1 \mathrm{~mm}^{2}\right)$ of regions containing glomeruli were specifically selected in the kidney. The tissue slices were prepared for TEM by following a previously described protocol [10]. In short, immediately after collection the specimens were immersed into a fixation buffer (2\% glutaraldehyde, $1 \%$ paraformaldehyde 
in $0.1 \mathrm{M}$ phosphate buffer) and placed in a refrigerator until further preparation. Prior to dehydration, the specimens were transferred to a wash buffer $(0.1 \mathrm{M}$ phosphate buffer) and placed in a refrigerator overnight. In order to increase the contrast of the tissue for TEM imaging, the samples were put in an Osmium fixation buffer $\left(2 \% \mathrm{OsO}_{4}\right.$ in $0.1 \mathrm{M}$ phosphate buffer). Thereafter, the samples were dehydrated in ethanol followed by acetone and embedded in Epoxy resin, LX 112. Ultra-thin sections of approximately $50 \mathrm{~nm}$ from the tissue slices were cut using a Leica Ultracut UCT (Leica, Austria) and placed on formvar coated copper grids coated with a thin carbon film. A total of 144 tissue sections, corresponding to 4 sections from each rat $(n=9)$ and tissue $(n=4)$, were prepared and imaged using a FEI CM 120 (Philips/FEI, The Netherlands) TEM at an accelerating voltage of $80 \mathrm{kV}$. Grids were excluded from the study if big cracks were found in the slices. Images were recorded onto Kodak SO-163 films and digitized using an Epson Perfection V600 photo scanner (Epson, US).

\section{Results}

\subsection{Ultrasound imaging}

\subsubsection{In-vitro: Lowest detectable concentration of contrast agent}

Concentrations higher than $10^{3} \mathrm{MBs} / \mathrm{ml}$ resulted in a significant difference between the mean intensity within $\mathrm{ROI}_{\text {control }}$ and $\mathrm{ROI}_{\text {dilution }}$ for both Plain MB and SonoVue, implying that the lowest detectable concentration was $10^{4} \mathrm{MBs} / \mathrm{ml}$ for both CAs.

\subsubsection{In-vivo: Blood elimination time measurements}

The wash-out curves for the CAs tested are shown in Fig. 4. Common for all CAs was a rapid decrease in the image intensity for all CAs in the early elimination phase. Nevertheless, a slight difference in intensity decrease pattern could be seen, whereas SonoVue and Type A had a more rapid intensity decrease in the early phase compared to Plain MB and Type B. On the other hand, all types of the novel CAs had significant longer detectable circulation times in blood than SonoVue, see Fig. 5 and Table 2. The blood half-life time for SonoVue was $7.5 \mathrm{~s}$, while the different polymer-shelled CAs have a blood half-life time of 17-85 s. When comparing the blood elimination time for the three types of the novel CA it can be concluded that Plain MB and Type B have a significant longer blood elimination time than Type A. There was no significant difference in blood elimination time for Plain MB and Type B.

\subsection{Histological analysis by transmission electron microscopy}

The MBs could clearly be differentiated from other subcellular structures since they have a sharp inner surface with a distinct shell structure. A total of $731 \mathrm{MBs}$ were found in all analyzed tissue sections and the MBs were exclusively found in the circulatory system, either taken up by, or found in the vicinity of or adherent to macrophages, see Fig. 6(A)-(F). No MBs uptake by parenchyma or endothelial cells was observed since all of the $731 \mathrm{MBs}$ found in the investigated organs were located inside the blood vessels. It was difficult to determine MB proximity to a specific cell type in the spleen, since there is a large variety of size and shape for each cell type. However, lysosomes could be seen close to where the MBs were found, indicating an uptake of MBs by macrophages also in the spleen, see Fig. 6(B) and (E). This problem was not observed for liver, kidney and lung tissue. As seen in Fig. 6(C), several MBs can be phagocytized by one single macrophage.

MBs of all types of the polymer-shelled CA, intact in the shape of spherical structures, were found in tissue sections at all collected time points. However, most MBs were found in tissue sections collected 


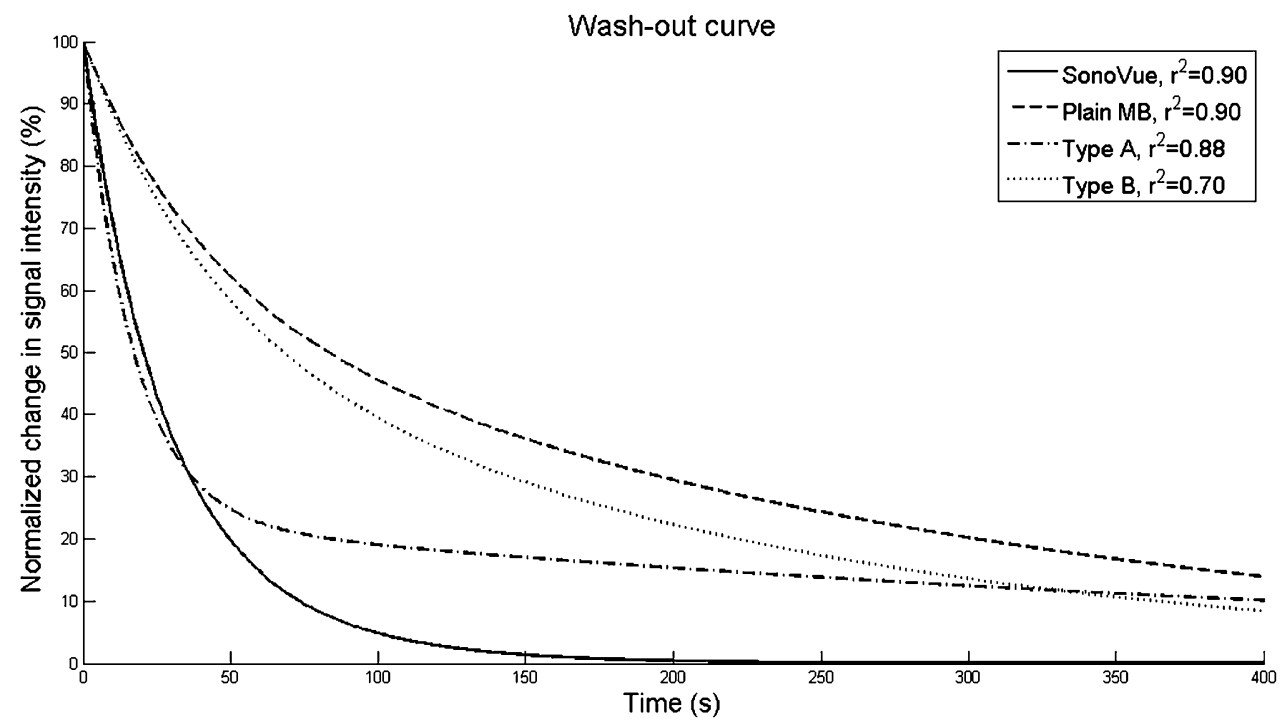

Fig. 4. Wash-out-curves obtained for the different contrast agents. SonoVue $(n=8)$ (solid), Plain MB $(n=7)($ dash-dash), Type A $(n=8)($ dash-dot) and Type $\mathrm{B}(n=7)$ (dotted), $r=$ correlation coefficient.
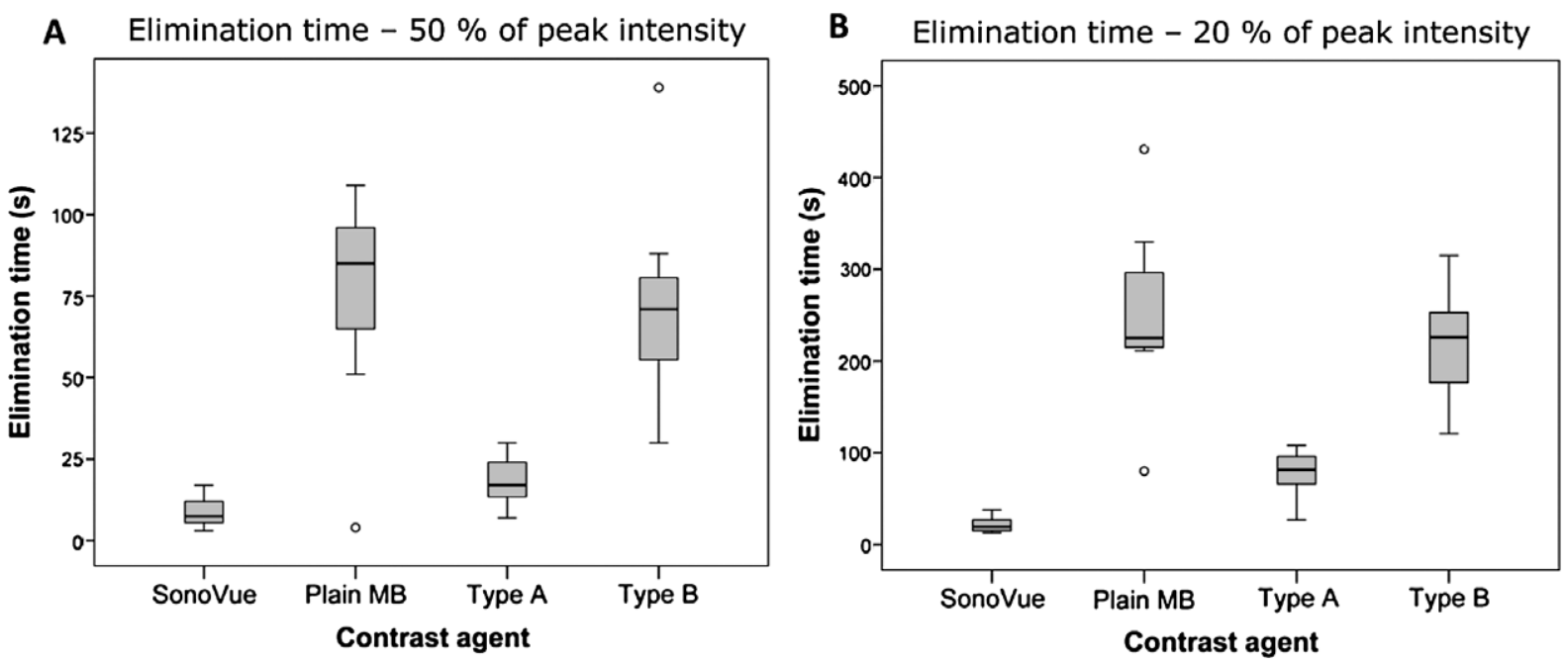

Fig. 5. Box plots showing the CA blood elimination times. Blood elimination times are shown at 50\% (A) and 20\% (B) of peak intensity for each contrast agent (SonoVue $(n=8)$, Plain MB $(n=7)$, Type A $(n=8)$ and type $\mathrm{B}(n=7))$ included in the study.

10 and 40 min after the injection, see Table 3. In addition, a few MBs, in particular Type A, had lost their spherical structure and it seems that they had collapsed, see Fig. 6(A). In tissue sections collected $24 \mathrm{~h}$ after the CA injection, SPIONs could still be seen attached to, see Fig. 6(E), or embedded inside the shell for Type A and Type B, respectively. In addition, even after $24 \mathrm{~h}$ of circulation the MBs were only found in blood vessels, close to or inside macrophages. Most of the MBs of both Type A and Type B were found in the lungs 10 and $40 \mathrm{~min}$ after injection. After $24 \mathrm{~h}$, a shift in the MB localization could be observed. Higher number of MBs were then located in the spleen and liver tissue sections compared 
Table 2

\begin{tabular}{|c|c|c|c|c|c|c|c|c|c|c|}
\hline \multirow[t]{3}{*}{$\overline{\mathrm{CA}}$} & \multirow{2}{*}{\multicolumn{2}{|c|}{ Elimination time (s) }} & \multicolumn{8}{|c|}{$P$-value } \\
\hline & & & \multicolumn{2}{|c|}{ SonoVue } & \multicolumn{2}{|c|}{ Plain MB } & \multicolumn{2}{|c|}{ Type A } & \multicolumn{2}{|c|}{ Type B } \\
\hline & $50 \%$ & $20 \%$ & $50 \%$ & $20 \%$ & $50 \%$ & $20 \%$ & $50 \%$ & $20 \%$ & $50 \%$ & $20 \%$ \\
\hline SonoVue & $\begin{array}{c}7.5 \\
(5.3-13.5)\end{array}$ & $\begin{array}{c}19.5 \\
(14-30)\end{array}$ & - & - & $P<0.001$ & $P<0.01$ & $P<0.05$ & $P<0.01$ & $P<0.001$ & $P<0.001$ \\
\hline Plain MB & $\begin{array}{c}85 \\
(51-104)\end{array}$ & $\begin{array}{c}225 \\
(211-330)\end{array}$ & $P<0.001$ & $P<0.01$ & - & - & $P<0.01$ & $P<0.01$ & NS & NS \\
\hline Type A & $\begin{array}{c}17 \\
(13.3-24)\end{array}$ & $\begin{array}{c}81.5 \\
(64.5-96.5)\end{array}$ & $P<0.05$ & $P<0.01$ & $P<0.01$ & $P<0.01$ & - & - & $P<0.001$ & $P<0.001$ \\
\hline Type B & $\begin{array}{c}71 \\
(54-88)\end{array}$ & $\begin{array}{c}226 \\
(146-258)\end{array}$ & $P<0.001$ & $P<0.001$ & NS & NS & $P<0.001$ & $P<0.001$ & - & - \\
\hline
\end{tabular}


Table 3

The number of microbubbles (MBs) localized in the tissue sections post contrast agent (CA) injection

\begin{tabular}{lcc}
\hline Time after CA injection & Number of analyzed tissue sections & Number of localized MBs \\
\hline $10 \mathrm{~min}$ & $42^{*}$ & 278 \\
$40 \mathrm{~min}$ & $46^{\ddagger}$ & 292 \\
$24 \mathrm{~h}$ & $46^{\ddagger}$ & 161 \\
\hline
\end{tabular}

* 6 grids were damaged during preparation and therefore excluded.

${ }^{\ddagger} 2$ grids were damaged during preparation and therefore excluded.

to previous time points. Few MBs, regardless of MB type and time after injection, were observed in the kidney. None of these MBs had passed the filtration barrier between blood and urine in the glomerulus. All MBs found in the kidney were located in the circulatory system, see Fig. 6(D).

\section{Discussion}

This study showed that the macrophage phagocytosis was the main responsible mechanism for eliminating the novel polymer-shelled CAs, since the MBs were exclusively found in the circulatory system, mainly taken up by, or found in the vicinity of or adherent to macrophages. None of the MBs were located outside the blood vessels, indicating that uptake in parenchyma or endothelial cells in these tissues can be excluded, or at least considered to be very low. Comparable results have also been reported using CA consisting of other shell compositions. For example, it was demonstrated that the macrophages were exclusively responsible for the uptake of lipid-shelled CA Sonazoid [17]. Additionally, it has been shown that macrophage cells were predominantly responsible for the uptake of iodine labeled albumin MBs [16]. However, it is important to remember that only a modest area from each tissue part and only a few animals were investigated in the histology analysis, implying that no quantitative measurements of the CA uptake per organ and CA type could be made using this technique. Nevertheless, none of the $731 \mathrm{MBs}$ localized in the analyzed tissues were seen leaving the blood vessels. Thus, this study indicates how the CA interacts on a subcellular level within the analyzed tissues. This is an advantage compared with other studies using indirect methods, such as tracing encapsulated gas [23] or measuring the radioactivity [25], since it is unknown how the encapsulated gas is released from the MBs and if the radiolabeled molecules are still attached to MBs after interaction with biological material. Hence, no information about both the MB structure and localization can be obtained using these other methods.

Considering the in-vitro experiments, the lowest detectable concentration of CA when using the present ultrasound system was $10^{4} \mathrm{MBs} / \mathrm{ml}$ for the CAs investigated. Variations in the lowest detectable concentration for the polymer CAs might be present, as the SPION coating partly affects the echogenicity response [19]. If assuming an even distribution of CA within the circulatory system after a few heart beats, the concentration of CA in blood post injection can be estimated to approximately $10^{7} \mathrm{MBs} / \mathrm{ml}$, considering the blood volume in the rats used for the ultrasound experiments to be approximately $28 \mathrm{ml}$ [21] and the fact that the injected dose was $0.4 \mathrm{ml}$ at a concentration of $6.6 \times 10^{8} \mathrm{MBs} / \mathrm{ml}$. This indicates that as low as one permille of the injected dose could approximately be detected using the present ultrasound system. Transferring this to the in-vivo environment, it should be kept in mind that the acoustic properties of the MBs can be altered by e.g. macrophages internalization of the MBs or by protein complexes surrounding the MBs in the blood, as further discussed below. The obtained blood elimination times might therefore, to some extent, differ from the true blood elimination time. 

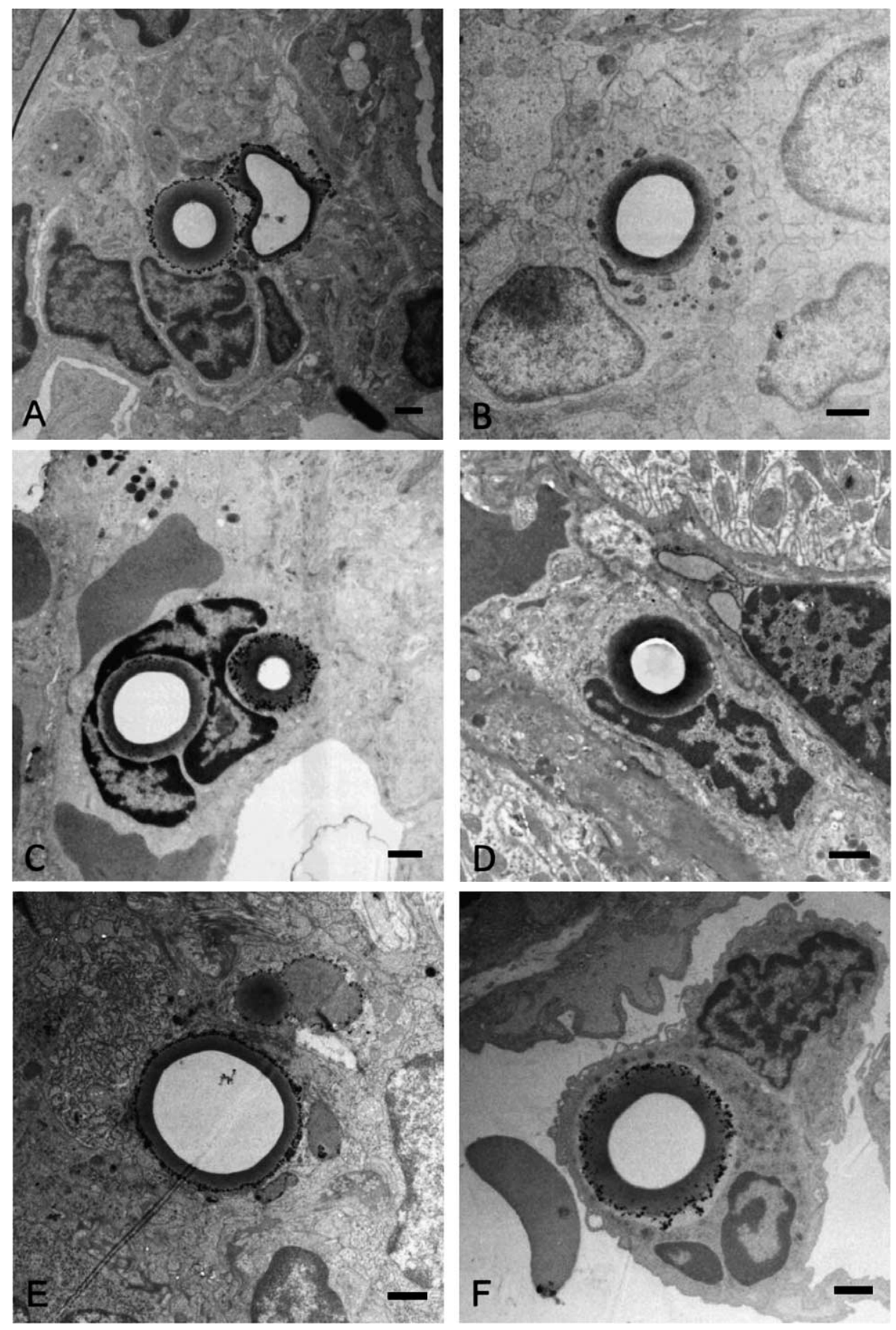

Fig. 6. TEM histology images of localized microbubbles. (A) Two Type A microbubbles (MBs), one intact (left) and one collapsed (right), found internalized in a rat lung macrophage 40 min post injection; (B) Plain MB found inside a rat spleen macrophage 40 min post injection; (C) Type B MBs observed in a rat lung macrophage 10 min post injection; (D) One plain MB inside a rat kidney macrophage $10 \mathrm{~min}$ post injection; (E) Type A MB observed in rat spleen macrophage $24 \mathrm{~h}$ post injection; (F) Type B MB internalized in a rat lung macrophage 40 min post injection. Scale bars represent $1 \mu \mathrm{m}$. 
The in-vivo study showed that all polymer-shelled CAs had significant longer detectable blood circulation times than SonoVue. However, Type A also showed a fast signal intensity decrease during the early elimination phase. Previous studies have shown that various coatings of iron oxide nanoparticles interact with different proteins in a biological solution and these proteins form complexes referred to as the protein corona [20]. The protein corona might affect the transportation across biological barriers [13]. Therefore, polymer-shelled MBs loaded with SPIONs might change the blood elimination profiles depending on its structure, in particular the outer surface that is facing the circulation system. In comparison to Plain MB and Type B, the outermost layer of Type A consists of SPIONs instead of polymers. Moreover, the shell of Type B has shown to be more rigid than the shell of Type A [3]. The difference in shell stiffness could answer why Type A has a higher tendency to collapse. These factors might explain the difference in the observed blood half-life for these CAs. However, the overall prolonged blood circulation time seen for polymer-shelled CAs offers more time for image acquisition, which enables the examination of organs in multiple scanning planes as well as contrast replenishment studies for quantitative assessment of perfusion with the use of one single bolus injection. Beside prolonged blood circulation time in the early elimination phase, intact MBs were still present in the blood circulation system $24 \mathrm{~h}$ after $\mathrm{CA}$ injection as seen in the TEM images. These findings were also obtained in a previous study, where the novel polymer-shelled CA was observed in organs $24 \mathrm{~h}$ post CA injection when using MRI [1]. Additionally, the shift of MBs from lungs $10 \mathrm{~min}$ post CA injection into the liver and spleen after $24 \mathrm{~h}$ has also been observed using another type of the polymer-shelled CA [2]. In both the short and the long time perspective, the prolonged blood elimination time is important for targeted imaging where long circulation time increases the probability of attachment of MBs to the intended targets. The ability of the imaging systems to detect single MBs is however crucial in this context.

The concentration of CA injected during histology analysis was approximately nine times lower than the one used during the ultrasound experiments. Thus, the phagocytosis process may have been affected when high concentration of MBs was injected due to saturation of the macrophages. Nevertheless, the observations obtained during the histology analysis indicated that several MBs can be phagocytized by one single macrophage. Moreover, assuming the calculations done in a previous study [17], the total number of liver macrophages will be in excess even if a dose above $10^{9} \mathrm{MBs} / \mathrm{ml}$ will be injected. Hence, the uptake in the macrophages will most probably not be affected if using comparable concentration for histology analysis as for ultrasound imaging.

In summary, the prolonged blood circulation times for the novel polymer-shelled CAs offers longer image acquisition times and possibilities to be used as vehicles for target imaging and drug delivery. To further understand the elimination process of the novel polymer-shelled CAs, quantitative measurements of the biodistribution and clearance must be performed. However, this study provides information about the elimination time in blood as well as knowledge about the subcellular localization of the three types of the novel CAs, thereby giving new insight of how these CAs are eliminated.

\section{Conclusions}

The elimination profile, determined by the use of ultrasound imaging, showed longer blood circulation time for the novel polymer-shelled CAs compared with the commercially available CA SonoVue. Plain MB and Type B had a significant longer blood circulation time than Type A. The macrophages were suggested to be responsible for the elimination of the different types of the novel CAs as MBs were exclusively found inside the circulatory system either taken up by, or found in the vicinity of or adherent to macrophages. 


\section{Acknowledgements}

This study was a part of the 3MiCRON (245572) project, which was founded by the European Commission within the Seventh Framework Program. We would like to acknowledge Dr. Kjell Hultenby and the staff of the Electron Microscopy Unit (EMil) at the Karolinska University Hospital, Huddinge, Sweden, for assistance with the preparation of tissue sections and valuable feedback when analyzing them. Support from the Center for Biosciences, Karolinska Institutet, Huddinge, Sweden is also acknowledged, as well as the Jonasson Center for Medical Imaging, Huddinge, Sweden.

\section{References}

[1] A. Barrefelt, T. Brismar, G. Egri, P. Aspelin, A. Olsson, L. Oddo, S. Margheritelli, K. Caidahl, G. Paradossi, L. Dahne et al., Multimodality imaging using SPECT/CT and MRI and ligand functionalized 99mTc-labeled magnetic microbubbles, EJNMMI Research 3(1) (2013), 12.

[2] A. Barrefelt, M. Saghafian, R. Kuiper, F. Ye, G. Egri, M. Klickermann, T.B. Brismar, P. Aspelin, M. Muhammed, L. Dahne et al., Biodistribution, kinetics, and biological fate of SPION microbubbles in the rat, Int. J. Nanomedicine 8 (2013), 32413254.

[3] T.B. Brismar, D. Grishenkov, B. Gustafsson, J. Harmark, A. Barrefelt, S.V. Kothapalli, S. Margheritelli, L. Oddo, K. Caidahl, H. Hebert et al., Magnetite nanoparticles can be coupled to microbubbles to support multimodal imaging, Biomacromolecules 13(5) (2012), 1390-1399.

[4] F. Cavalieri, A. El Hamassi, E. Chiessi and G. Paradossi, Stable polymeric microballoons as multifunctional device for biomedical uses: synthesis and characterization, Langmuir 21(19) (2005), 8758-8764.

[5] F. Cavalieri, A. El Hamassi, E. Chiessi, G. Paradossi, R. Villa and N. Zaffaroni, Tethering functional ligands onto shell of ultrasound active polymeric microbubbles, Biomacromolecules 7(2) (2006), 604-611.

[6] J.M. Correas, A.R. Meuter, E. Singlas, D.R. Kessler and D. Worah, Quay SC: human pharmacokinetics of a perfluorocarbon ultrasound contrast agent evaluated with gas chromatography, Ultrasound Med. Biol. 27(4) (2001), 565-570.

[7] C. Greis, Technology overview: SonoVue (Bracco, Milan), Eur. Radiol. 14(Suppl. 8) (2004), P11-P15.

[8] D. Grishenkov, C. Pecorari, T.B. Brismar and G. Paradossi, Characterization of acoustic properties of PVA-shelled ultrasound contrast agents: ultrasound-induced fracture (Part II), Ultrasound Med. Biol. 35(7) (2009), 1139-1147.

[9] D. Grishenkov, C. Pecorari, T.B. Brismar and G. Paradossi, Characterization of acoustic properties of PVA-shelled ultrasound contrast agents: linear properties (Part I), Ultrasound Med. Biol. 35(7) (2009), 1127-1138.

[10] A. Hansson, N. Hance, E. Dufour, A. Rantanen, K. Hultenby, D.A. Clayton, R. Wibom and N.G. Larsson, A switch in metabolism precedes increased mitochondrial biogenesis in respiratory chain-deficient mouse hearts, Proc. Natl. Acad. Sci. USA 101(9) (2004), 3136-3141.

[11] J.R. Harris, F. Depoix and K. Urich, The structure of gas-filled $n$-butyl-2-cyanoacrylate (BCA) polymer particles, Micron 26(2) (1995), 103-111.

[12] J.C. Hutter, H.M.D. Luu, P.M. Mehlhaff, A.L. Killam and H.C. Dittrich, Physiologically based pharmacokinetic model for fluorocarbon elimination after the administration of an octafluoropropane-albumin microsphere sonographic contrast agent, J. Ultrasound Med. 18(1) (1999), 1-11.

[13] A. Jedlovszky-Hajdu, F.B. Bombelli, M.P. Monopoli, E. Tombacz and K.A. Dawson, Surface coatings shape the protein corona of SPIONs with relevance to their application in vivo, Langmuir 28(42) (2012), 14983-14991.

[14] J. Kamps, H.W.M. Morselt, P.J. Swart, D.K.F. Meijer and G.L. Scherphof, Massive targeting of liposomes, surfacemodified with anionized albumins, to hepatic endothelial cells, Proc. Natl. Acad. Sci. USA 94(21) (1997), 11681-11685.

[15] A.L. Killam, P.M. Mehlhaff, P.A. Zavorskas, Y. Greener, B.A. McFerran, J.J. Miller, C. Burrascano, E.G. Jablonski, L. Anderson and H.C. Dittrich, Tissue distribution of I-125-labeled albumin in rats, and whole blood and exhaled elimination kinetics of octafluoropropane in anesthetized canines, following intravenous administration of OPTISON (R) (FS069), Int. J. Toxicol. 18(1) (1999), 49-63.

[16] G.M. Kindberg, H. Tolleshaug, T. Gjoen and T. Berg, Lysosomal and endosomal heterogeneity in the liver: a comparison of the intracellular pathways of endocytosis in rat liver cells, Hepatology 13(2) (1991), 254-259.

[17] G.M. Kindberg, H. Tolleshaug, N. Roos and T. Skotland, Hepatic clearance of Sonazoid perfluorobutane microbubbles by Kupffer cells does not reduce the ability of liver to phagocytose or degrade albumin microspheres, Cell Tissue Res. 312(1) (2003), 49-54.

[18] M. Lampaskis and M. Averkiou, Investigation of the relationship of nonlinear backscattered ultrasound intensity with microbubble concentration at low MI, Ultrasound Med. Biol. 36(2) (2010), 306-312. 
[19] M. Larsson, M. Larsson, L. Oddo, S. Margheritelli, G. Paradossi, J. Nowak, L.A. Brodin, K. Caidahl and A. Bjallmark, Visualization of multimodal polymer-shelled contrast agents using ultrasound contrast sequences: an experimental study in a tissue mimicking flow phantom, Cardiovasc. Ultrasound 11(1) (2013), 33.

[20] L. Lartigue, C. Wilhelm, J. Servais, C. Factor, A. Dencausse, J.C. Bacri, N. Luciani and F. Gazeau, Nanomagnetic sensing of blood plasma protein interactions with iron oxide nanoparticles: impact on macrophage uptake, ACS Nano 6(3) (2012), $2665-2678$.

[21] H.B. Lee and M.D. Blaufox, Blood-volume in the rat, J. Nucl. Med. 26(1) (1985), 72-76.

[22] D.R. Morel, I. Schwieger, L. Hohn, J. Terrettaz, J.B. Llull, Y.A. Cornioley and M. Schneider, Human pharmacokinetics and safety evaluation of SonoVue (TM), a new contrast agent for ultrasound imaging, Invest. Radiol. 35(1) (2000), 80-85.

[23] K.G. Toft, S.O. Hustvedt, P.A. Hals, I. Oulie, S. Uran, K. Landmark, P.T. Normann and T. Skotland, Disposition of perfluorobutane in rats after intravenous injection of Sonazoid (TM), Ultrasound Med. Biol. 32(1) (2006), 107-114.

[24] P. Walday, H. Tolleshaug, T. Gjoen, G.M. Kindberg, T. Berg, T. Skotland and E. Holtz, Biodistributions of air-filled albumin microspheres in rats and pigs, Biochem. J. 299 (1994), 437-443.

[25] J.K. Willmann, Z. Cheng, C. Davis, A.M. Lutz, M.L. Schipper, C.H. Nielsen and S.S. Gambhir, Targeted microbubbles for imaging tumor angiogenesis: assessment of whole-body biodistribution with dynamic micro-PET in mice, Radiology 249(1) (2008), 212-219. 